Wavenumber Selection by Bénard-Marangoni Convection at High Supercritical Number

This content has been downloaded from IOPscience. Please scroll down to see the full text.

2017 Chinese Phys. Lett. 34054702

(http://iopscience.iop.org/0256-307X/34/5/054702)

View the table of contents for this issue, or go to the journal homepage for more

Download details:

IP Address: 159.226.231.78

This content was downloaded on 25/07/2017 at 03:31

Please note that terms and conditions apply.

You may also be interested in:

Oscillatory and Chaotic Buoyant-Thermocapillary Convection in the Large-Scale Liquid Bridge Jia Wang, Li Duan and Qi Kang

Longwave oscillatory patterns in liquids: outside the world of the complex Ginzburg-Landau equation Alexander Nepomnyashchy and Sergey Shklyaev

Longwave convection in a layer of binary mixture with modulated heat flux: weakly nonlinear analysis

Irina S Fayzrakhmanova, Sergey Shklyaev and Alexander A Nepomnyashchy

Mechanism of mode selection in Rayleigh-Bénard convection with free-rigid boundaries

Jiro Mizushima

Formation of spatial structures in Rayleigh-Bénard convection

Aleksandr V Getling

Longwave Marangoni convection in a binary liquid layer heated from above: weakly nonlinear analysis Sergey Shklyaev and Alexander A Nepomnyashchy

Generation of transverse waves in a liquid layer with insoluble surfactant subjected to temperature gradient

Alexander B Mikishev, Barry A Friedman and Alexander A Nepomnyashchy

Instability of Two-Layer Rayleigh-Bénard Convection withInterfacial Thermocapillary Effect

Liu Qiu-Sheng, Zhou Bing-Hong, Nguyen Thi Henri et al.

Transport phenomena and dimensionless numbers: towards a new methodological approach

B A Bezuglyi, N A Ivanova and L V Sizova 


\title{
Wavenumber Selection by Bénard-Marangoni Convection at High Supercritical Number *
}

\author{
$\operatorname{Di} \mathrm{Wu}(\text { 吴笛 })^{1}, \mathrm{Li} \operatorname{Duan}\left(\mathbf{E}_{\mathbf{Z}} \text { 例 }\right)^{1,2 * *}$, Qi Kang(康琦) $)^{1,2 * *}$ \\ ${ }^{1}$ Key Laboratory of Microgravity (National Microgravity Laboratory), Institute of Mechanics, \\ Chinese Academy of Sciences, Beijing 100190 \\ ${ }^{2}$ School of Engineering Science, University of Chinese Academy of Sciences, Beijing 100049
}

(Received 13 November 2016)

\begin{abstract}
Marangoni-Bénard convection, which is mainly driven by the thermocapillary (Marangoni) effect, occurs in a thin liquid layer heated uniformly from the bottom. The wavenumber of supercritical convection is studied experimentally in a $160 \times 160-\mathrm{mm}^{2}$ cavity that is heated from the bottom block. The convection pattern is visualized by an infrared thermography camera. It is shown that the onset of the Bénard cell is consistent with theoretical analysis. The wavenumber decreases obviously with increasing temperature, except for a slight increase near the onset. The wavenumber gradually approaches the minimum when the supercritical number $\varepsilon$ is larger than 10. Finally, a formula is devised to describe the wavenumber selection in supercritical convection.
\end{abstract}

PACS: 47.20.Dr, 47.54.-r, 47.54.De $\quad$ DOI: 10.1088/0256-307X/34/5/054702

Hexagonal convective cells emerge in a thin liquid layer if the temperature difference in the vertical direction exceeds the threshold. ${ }^{[1]}$ This is a classical phenomenon in hydrodynamics, called the Bénard instability, which is mainly caused by the thermocapillary effect (Marangoni effect). The Bénard convection has been applied in many industrial processes, such as the film and coating industries. Controlling cell size is of great importance in manufacturing a self-organized structure, e.g., in honeycomb-patterned porous films ${ }^{[2]}$ or in hexagonal nanocrystals. ${ }^{[3]}$ However, how a wavenumber is selected by the Bénard-Marangoni convection has yet to be determined.

Wavenumber, which is inversely proportional to the cell size, is selected by the temperature difference across the layer. Near the onset, the hexagonal cell is organized by a single critical wavenumber $k_{\mathrm{c}}$. In the supercritical case, on the other hand, the nonlinear competition of wave disturbances in an unstable band makes the wavenumber selection difficult to solve. Cloot and Lebon ${ }^{[4]}$ predicted that the supercritical wavenumbers in the pure Marangoni convection are larger than the critical wave number, and the supercritical wavenumbers under the coupling of buoyancy and thermocapillary force $(400<R a<500)$ are smaller than the critical wave number. Bestehorn ${ }^{[5]}$ described a stable band with a hexagonal pattern by phase and amplitude stability analysis that increases near the threshold, and also found Kosechmieder's experimental results ${ }^{[6]}$ located in their expected band. However, the decrease of wavenumber beyond the threshold in experiments cannot be explained.

As a dissipative structure, the number of cells is expected to increase to dissipate more energy in the supercritical case. Because of strong nonlinear ef- fects, the wavenumber decreases monotonically with the temperature difference in a buoyancy convection cell. Koschmieder et al. ${ }^{[6]}$ found a more complex selection of the Marangoni-Bénard convection, in which the wavenumber slightly increases near threshold and then decreases beyond it. However, only the region in which $\Delta T<3 \Delta T_{\mathrm{c}}$ is covered in their experiment, whereas the mechanism by wavenumber is selected in the region far beyond the threshold is still undetermined.

In this Letter, we study the wavenumber selection by the supercritical number in the Marangoni-Bénard convection away from the threshold. The Fourier analysis of the surface temperature field can reveal the convective pattern wavenumber, because the thermocapillary flow is determined by the surface temperature distribution. The preferred wavenumber in a real physical system is in a rather narrow band, and therefore we consider the average wavenumber of the band as the selected wavenumber for our discussion. Experiments in different test fluids and at different liquid thicknesses are conducted to study the strong nonlinear selection of the wavenumber.

All of the experiments were carried on a $160 \times 160$ $\mathrm{mm}^{2}$ heating cavity (see Fig. 1), the rigid walls were fabricated with K9 glasses. The heating plate was made from a sandwich structure of a copper block, an aluminum block, and a silica gel layer, which provides good temperature uniformity. The bottom plate was heated by an electric heating film and the temperature was controlled by a Eurotherm module (Model No. 3306) with $0.01{ }^{\circ} \mathrm{C}$ fluctuation. We linearly increased the temperature at a slow ramp of $0.2^{\circ} \mathrm{C} / \mathrm{min}$ to observe the steady-state convection. To cover the strongly nonlinear convection in the experiment, the maximum

* Supported by the National Natural Science Foundation of China under Grant Nos 11502271 and 11372328 , the Strategic Priority Research Program on Space Science of the Chinese Academy of Sciences under Grant Nos XDA04020405 and XDA04020202-05, and the China Manned Space Engineering Program.

** Corresponding author. Email: duanli@imech.ac.cn; kq@imech.ac.cn

(C) 2017 Chinese Physical Society and IOP Publishing Ltd 
temperature approached $70^{\circ} \mathrm{C}$, at which point the supercritical number reached approximately 30 for some low-viscosity convection.

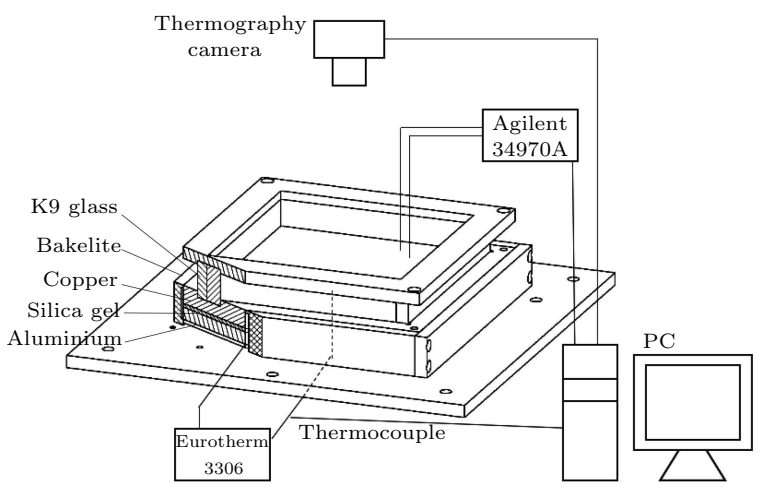

Fig. 1. Schematic diagram of the experimental apparatus.

The convection cell is visualized from the top of the liquid layer by an infrared thermography camera (FLIR Model No. E60, FLIR Systems, Inc., USA). The observed region in the middle of the cavity was measured to be approximately $98 \times 86 \mathrm{~mm}^{2}$. The temperature difference was measured via two T-type thermocouples, one fixed at the bottom of the layer and the other at the top. The temperature was recorded by a data acquisition/switch unit (Agilent Model No. 34970A, Agilent Technologies, USA). It is noticed that the position of the top thermocouple should be adjusted during the experiment because the surface rises due to the thermal expansion of the bottom block and the liquid layer.

It is well known that two different mechanisms account for the Bénard convection, i.e., surface tension and buoyancy force. Surface-tension-driven convection occurs in a very thin layer or in a microgravity circumstance, and is called the Bénard-Marangoni convection. ${ }^{[8]}$ Otherwise, buoyancy-driven convection occurs in a thick layer without a free surface, and is called the Rayleigh-Bénard convection. ${ }^{[9]}$ The driven forces of the two types of convection are nondimensionalized as the $M a$ number and the $R a$ number, respectively. Moreover, two other important dimensionless parameters that affect the convection are the $P r$ number and the $B i$ number. The $P r$ number characterizes the effect of liquid physical properties and the $B i$ number characterizes the heat transfer capacity of the surface. These numbers read

$$
\begin{aligned}
M a & =\frac{(\partial \sigma / \partial T) d \Delta T}{\rho \nu \kappa}, \quad R a=\frac{\alpha g d^{3} \Delta T}{\nu \kappa}, \\
\operatorname{Pr} & =\frac{\nu}{\kappa}, \quad B i=\frac{\alpha d}{\lambda},
\end{aligned}
$$

where $\rho, \kappa, \alpha, \nu, \partial \sigma / \partial T, g, d$, and $\Delta T$ are the density, thermal diffusivity, thermal expansion, kinematics viscosity, temperature coefficient of surface tension, magnitude of gravity, thickness of the liquid, and the temperature difference across the liquid, respectively.

Several kinds of silicone oil KF96 (manufactured by Shin-Etsu Chemical Co., Ltd.) ${ }^{[10]}$ were employed in the experiments. Their kinematics viscosities are $1.5,2,5,10$, and $50 \mathrm{cSt}$, the $\operatorname{Pr}$ numbers are 16.16, $25.21,62.5,113.38$, and 467.29 , respectively. The physical properties of these oils are listed in Table 1 . We estimate the $B i$ number to be approximately $0.2-0.3$ for the natural convective heat transfer of the surface. To focus on the predominately thermocapillary convection, experiments were carried out on thin layers $(d<3 \mathrm{~mm})$ because $R a\left(\sim O\left(d^{3}\right)\right)$ can be neglected compared with $M a(\sim O(d))$ for small values of $d$.

Table 1. Physical properties of KF96 silicone oil used in experiments.

\begin{tabular}{cccllll}
\hline Oil type & $\rho\left(\mathrm{kg} / \mathrm{m}^{3}\right)$ & $C_{\mathrm{p}}(\mathrm{J} / \mathrm{kg} \cdot \mathrm{K})$ & $\kappa\left(\mathrm{m}^{2} / \mathrm{s}\right)$ & $\alpha\left(\mathrm{K}^{-1}\right)$ & $\nu\left(\mathrm{m}^{2} / \mathrm{s}\right)$ & $\partial \sigma / \partial T(\mathrm{~N} / \mathrm{m} \cdot \mathrm{K})$ \\
\hline KF96-1.5 & 852 & 1974 & $0.595 \times 10^{-7}$ & $1.27 \times 10^{-3}$ & $1.5 \times 10^{-6}$ & \\
KF96-2 & 873 & 1764 & $0.714 \times 10^{-7}$ & $1.24 \times 10^{-3}$ & $2 \times 10^{-6}$ & $-4.6 \times 10^{-5}$ \\
KF96-5 & 915 & 1764 & $0.8 \times 10^{-7}$ & $1.09 \times 10^{-3}$ & $5 \times 10^{-6}$ & $-6.37 \times 10^{-5}[7]$ \\
KF96-10 & 935 & 1680 & $0.882 \times 10^{-7}$ & $1.06 \times 10^{-3}$ & $10 \times 10^{-6}$ & $-3.75 \times 10^{-5}$ \\
KF96-50 & 960 & 1512 & $1.07 \times 10^{-7}$ & $0.96 \times 10^{-3}$ & $50 \times 10^{-6}$ & $-4.7 \times 10^{-5}$ \\
\hline
\end{tabular}

Table 2. Thresholds of the convection onset.

\begin{tabular}{ccccc}
\hline Thickness $(\mathrm{mm})$ & 1.500 & 1.913 & 2.245 & 2.980 \\
\hline$T_{\mathrm{c}}\left({ }^{\circ} \mathrm{C}\right)$ & 4.74 & 4.14 & 3.34 & 2.52 \\
$M a_{\mathrm{c}}$ & 96.62 & 93.63 & 82.53 & 79.29 \\
$R a_{\mathrm{c}}$ & 42.63 & 67.20 & 81.56 & 138.07 \\
$k_{\mathrm{c}}$ & 1.966 & 1.989 & 1.994 & 2.077 \\
\hline
\end{tabular}

Taking the 50-cSt silicone oil layer with different thicknesses, the thickness of the liquid was measured using a displacement stage with a precision of $1 \mu \mathrm{m}$. The threshold of the convection onset for different thicknesses is listed in Table 2. The nonBoussinesq effect of the decreasing viscosity with temperature is taken into consideration by the formula $\log \nu_{T}=\frac{763.1}{273+T}-2.559+\log \nu_{25} \cdot{ }^{[10]}$ By considering the variation of viscosity with temperature, the on- set is found to fit better with Nield's theory, which predicts that $M a_{\mathrm{c}}$ decreases and $R a_{\mathrm{c}}$ increases with the layer thickness. ${ }^{[11]}$ The $M a_{\mathrm{c}}$ values listed in Table 2 are calculated using $\nu_{T}$. Koschmieder et al. ${ }^{[6]}$ gave $M a_{\mathrm{c}}=72.1$ in the 1.90 -mm layer without considering such non-Boussinesq effects. Our result will be consistent with theirs if a constant viscosity $\nu_{25}$ is used, which gives $M a_{\mathrm{c}}=72.5$ in the $1.913-\mathrm{mm}$ layer.

The critical wave $k_{\mathrm{c}}$ is calculated from the twodimensional (2D) Fourier spectrum. Taking the hexagonal pattern as an example, it can be considered as the superposition of three disturbance waves at the angle of $120^{\circ}$. Specifically, Fig. 2 shows the temperature distribution of the hexagonal Bénard cells captured by the thermography camera and its 2D Fourier 
spectrum. There are three waves in the spectrum, marked by squares labeled by a, b and c, and their inverse Fourier transforms are shown in Figs. 3(a)$3(\mathrm{c})$, respectively. Interestingly, the superimposition of strips in Figs. 3(a)-3(c) gives the hexagonal pattern in Fig. 3(d).

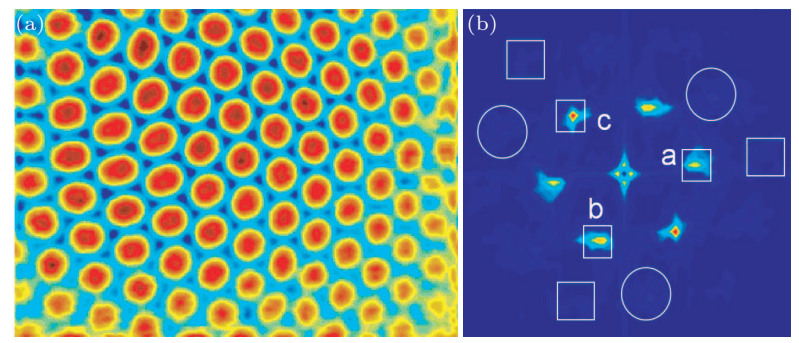

Fig. 2. (a) Hexagonal cells, and (b) 2D Fourier spectra.

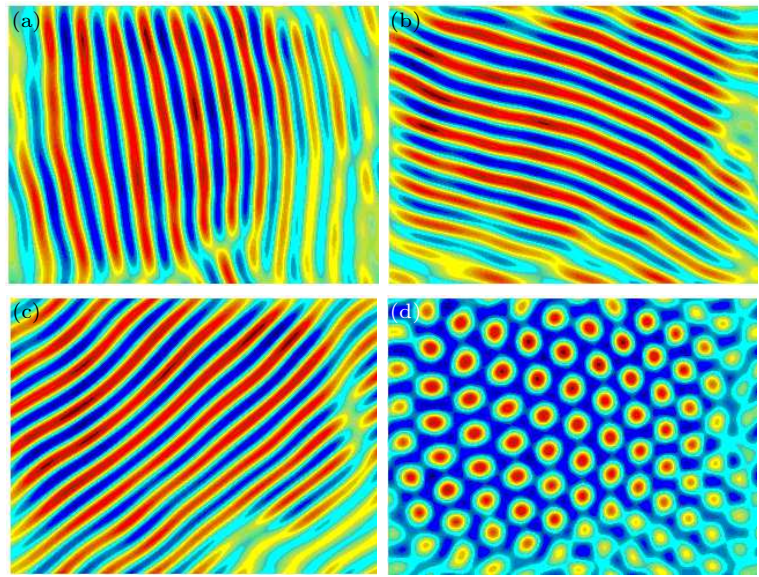

Fig. 3. (a)-(c) Inverse 2D Fourier transformation of waves a, b and c, respectively, of the spectra shown in Fig. 2(b). (d) Inverse 2D Fourier spectrum of the superimposition of waves a, b and c marked in Fig. 2(b).

The figures demonstrate that these waves, designated excited waves, determine the scale of the convective pattern. The relation between the wavenumber $k$ and the cell size $L$ is $k=4 \pi d / 3 L$. $^{[6]}$ It should be noted that the wavenumber must be nondimensionalized by thickness. Moreover, the effect of thermal expansion of the liquid layer is considered to improve the accuracy of wavenumber calculation.

Wavenumber selection by the supercritical number for different liquid thicknesses is presented in Fig. 4. With increasing $\varepsilon$, the wavenumber first increases and then decreases. The critical wavenumbers at the onset $\varepsilon=0$ are listed in Table 2, and they are close to the result from linear analysis, $k_{\mathrm{c}}=1.993$. An intuitive supercritical extrapolation is to assume that the fastest-growing wave is preferred in real convection. However, this assumption is tenable only in the region very near threshold $(\varepsilon<0.1)$, and it cannot explain the decrease of the wavenumber when $\varepsilon>0.6$. The reversal of wavenumber change rate probably involves some complex nonlinear effects.

The rigid sidewalls may also select the wavenumber, because they may shrink the instability wave band to a discrete value, which is proportional to $\sqrt{\varepsilon}$ in an infinite system. According to Cerisier et al. ${ }^{[12]}$ if $\Gamma>70$, the layer is large enough to neglect the influence of the sidewalls. The different thicknesses plotted in Fig. 4., i.e., 1.5, 1.9, 2.2 and $3.0 \mathrm{~mm}$, correspond to aspect ratios $\Gamma=107,84,70$, and 53, respectively. No distinct influence of thickness can be found when $d \leq 2.2 \mathrm{~mm}(\Gamma \geq 70)$. However, it is worth noting that the sidewalls confine the wavenumber variation in the layer with $d=3.0 \mathrm{~mm}(\Gamma>70)$, which behaves differently from that with $d \leq 2.2 \mathrm{~mm}$. To exclude the effect of sidewalls, experiments should be conducted at the layer with $d \leq 2.2 \mathrm{~mm}$ in our cavity.

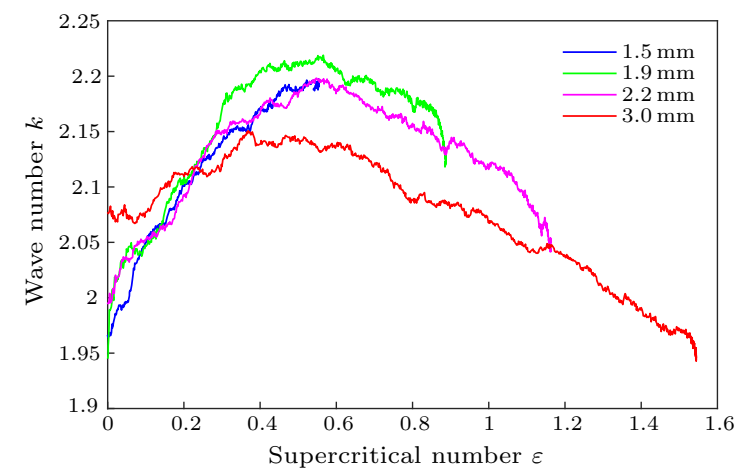

Fig. 4. Wavenumber selection with supercritical number for 50-cSt silicone oil.

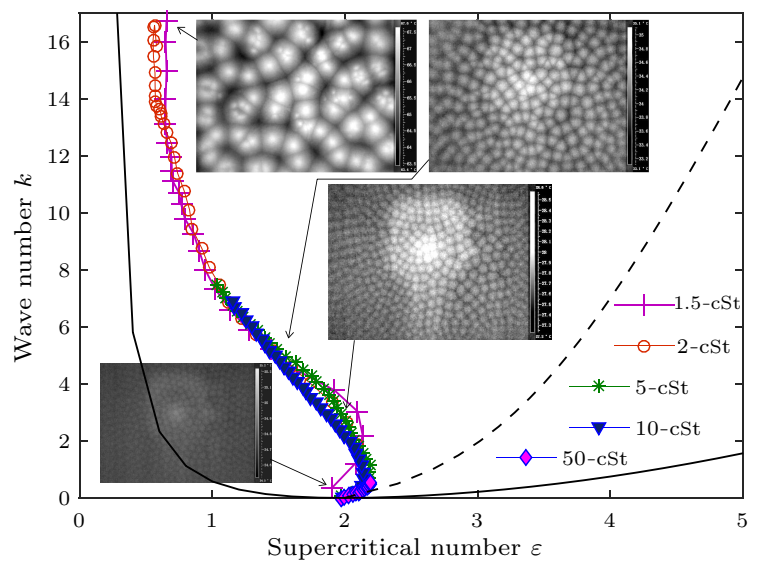

Fig. 5. Wavenumber selection at high supercritical number. The dashed and solid lines denote the fastest-growing wave and neutral stability curve, respectively.

To observe the strong nonlinear effect, highly supercritical convection is achieved by increasing the temperature difference and by choosing the lowviscosity liquid. The results for 1.5-, 2-, 5-, 10-, and 50-cSt silicone oil of $1.5 \mathrm{~mm}$ thickness are presented in Fig. 5. It is found that the wavenumber continuously decreases at large $\varepsilon$, except for a slight increase near the threshold. The wavenumber gradually approximates the minimum wavenumber $(0.56)$ after $\varepsilon>10$. This means that the maximum cell size is 3.56 times larger than the critical size.

Figure 5 shows some typical patterns of transformation in 2-cSt silicone oil of $1.5 \mathrm{~mm}$ thickness. Glo- 
bal convection occurs when $\varepsilon=0$ in this experiment. The transition from hexagonal to square cell is found at $\varepsilon=2.28$, which is consistent with the secondary instability found by Nitschke et al. ${ }^{[13]}$ at $\varepsilon=2.35$. When $\varepsilon=5.50$, the convection was dominated by irregular patterns instead of square patterns. When $\varepsilon=11.4$, the cell becomes very large and subcells start to emerge, which mean that the energy of smallscale disturbances grows by strongly nonlinear action. Subcells are periodically generated and diminished, which makes the flow time dependent. Extremely large cells are formed when $\varepsilon=16.6$. The enlargement of the cell is probably prevented by the action of the subcell, which breaks the larger cell into small subcells.

Kosechmieder et al. ${ }^{[6]}$ attributed the decrease of the wavenumber to the buoyancy effect, based on the prediction of Cloot et al. ${ }^{[4]}$ However, if so, it will not seem very persuasive that we should neglect the buoyancy at onset. From our perspective, the convection probably tends to select the wave that maximizes the convective energy. Consequently, the fastest-growing wave, indicated by a dashed line in Fig. 5, is selected near the threshold. In the supercritical case, the band above the neutral stability curve (the solid line in Fig. 5) is unstable. A small excitation wavenumber can keep more harmonic or passive wave in the band, which has the benefit that the small scale (passive wave) gains more energy. Therefore, the wavenumber decreases and is very close to the minimum wave in the band.

To the best of our knowledge, an empirical formula for wavenumber selection has not been given by other researchers. The conjecture that $k \sim 1 / \sqrt{M a^{[14]}}$ deviates from experiment, which misfits an increasing wavenumber near the threshold. We suppose that wavenumber selection satisfies the following formula

$$
k=\left(k_{\mathrm{c}}-k_{\infty}\right) \sqrt{(1+a \varepsilon)} e^{-b \varepsilon}+k_{\infty},
$$

where $k(\varepsilon) \sim \sqrt{(1+a \varepsilon)}$ when $\varepsilon \approx 0$, and $k(\varepsilon)$ decreases exponentially to $k_{\infty}$ when $\varepsilon \rightarrow \infty$.

According to the experimental result, the critical wavenumber is $\lim _{\varepsilon \rightarrow 0} k=k_{\mathrm{c}}=1.99$ and the saturated wavenumber is $\lim _{\varepsilon \rightarrow \infty} k=k_{\infty}=0.56$. Comparing Eq. (2) with the experimental result, we find that the formula fits the experiment very well when $a=3 / 2$ and $b=1 / 3$, as shown in Fig. 6 . Though without a rigorous proof, the empirical formula presented above can provide an estimation of wavenumber selection in industrial applications.

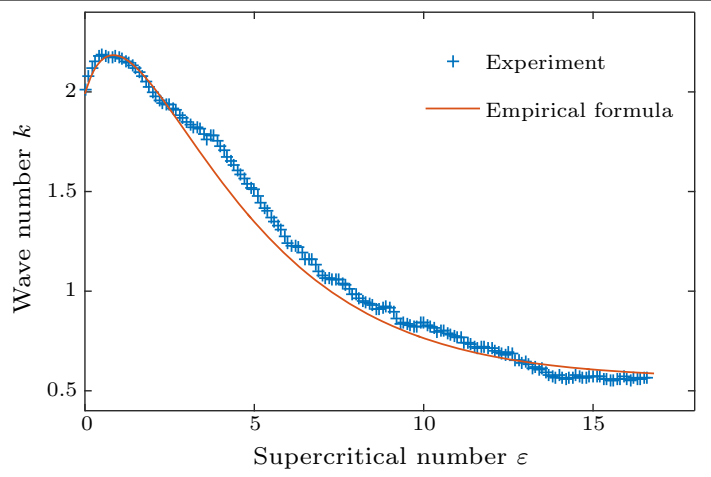

Fig. 6. Average wavenumber selection in experiments and empirical curve.

In summary, an experimental study of wavenumber selection has been carried out on the MarangoniBénard convection. When the aspect ratio $\Gamma>70$, the wavenumber selection curve was not affected by the sidewalls. The wavenumber slightly increases near the onset and decreases obviously with the supercritical number. We conjecture that the wavenumber selection tends to maximize the convective energy. Therefore, the fastest-growing waves are selected near the threshold and small wavenumbers, for which harmonic waves can gain more energy, are preferred. The wavenumber gradually approximates to the minimum value, $k=0.56$, instead of 0 for large supercritical numbers. This is because the enlargement of the cells stops after the emergence of subcells. The $\operatorname{Pr}$ number is also found to have no considerable effect on the wavenumber selection. Finally, we present an empirical formula for the wavenumber selection in a thin layer of Bénard convection.

\section{References}

[1] Bernard H 1900 Rev. Gén Sci. Pure Appl. 111261

[2] Widawski G, Rawiso M and François B 1994 Nature 369 387

[3] Maillard M, Motte L and Pileni M 2001 Adv. Mater. 13 200

[4] Cloot A and Lebon G 1984 J. Fluid Mech. 145447

[5] Bestehorn M 1993 Phys. Rev. E 483622

[6] Koschmieder E L and Switzer D W 1992 J. Fluid Mech. 240533

[7] Shevtsova V, Mialdun A, Kawamura H, Ueno I, Nishino K and Lappa M 2011 Fluid Dyn. Mater. Process. 71

[8] Pearson J R A 1958 J. Fluid Mech. 4489

[9] Rayleigh L 1916 Philos. Mag. 32529

[10] Silicone Fluid Kf-96 Performance Test Results 2008 (ShinEtsu Chemlcal Co., Ltd. Japan)

[11] Nield D A 1964 J. Fluid Mech. 19341

[12] Cerisier P, Perezgarcia C, Jamond C and Pantaloni J 1987 Phys. Rev. A 351949

[13] Nitschke K and Thess A 1995 Phys. Rev. E 52 R5772

[14] Moriarty P, Taylor M D R and Brust M 2002 Phys. Rev. Lett. 89248303 